Trace Regularity for a Second Order Hyperbolic Equation with Nonsmooth Coefficients

Gang Bao

and

William W. Symes

January, 1991

TR91-1 



\title{
TRACE REGULARITY FOR A SECOND ORDER HYPERBOLIC EQUATION WITH NONSMOOTH COEFFICIENTS ${ }^{1}$
}

\author{
Gang Bao and William W. Symes \\ Department of Mathematical Sciences \\ Rice University \\ Houston, Texas 77251-1892
}

\begin{abstract}
In this research, a trace regularity theorem on a time like surface is proved for the solution of a multiclimensional linear acoustic wave equation with nonsmooth coefficients. Our theorem indicates that with microlocal restrictions against tangential oscillations in the coefficients, the boundary value is just as regular as the solution, in particular as regular as the coefficients allow.
\end{abstract}

Key words. trace theorem, microlocal Sobolev spaces, propagation of singularities, pseudo-differential cut-off.

Abbreviated title: TRACE REGULARITY

\footnotetext{
${ }^{1}$ This work was partially supported by the National Science Foundation under grant DMS 86-03614 and DMS 89-05878, by the Office of Naval Research under contracts N00014-K-85-0725 and N00014-J-89-1115, by AFOSR 89-0363, and by the Geophysical Parallel Computation Project (State of Texas).
} 



\section{Introduction}

The classical trace theorem in Sobolev spaces asserts that the restriction map of a distribution to a codimension one hypersurface extends uniquely to a continuous linear operator from $H^{s}\left(\mathbb{R}^{k}\right)$ to $H^{s-1 / 2}\left(\mathbb{R}^{k-1}\right)$, if $s>1 / 2$. It is also well known that this result is sharp, see Taylor [18] for details. However, dealing with the solutions to hyperbolic p.d.e., one may reasonably expect an improvement of their trace regularity. This is actually the case if the equation with smooth coefficients is strictly hyperbolic with respect to a codimension one trace hypersurface, since then standard energy estimates will yield that the trace map is from $H^{s}\left(\mathbb{R}^{k}\right)$ to $H^{s}\left(\mathbb{R}^{k-1}\right)$ locally for any real $s$. Unfortunately, the same idea will not work if the trace surface is timelike, essentially because the presence of grazing rays prohibit the direct application of energy estimates. See Symes [16] and Bao and Symes [2] for more comments on this aspect. It is obvious that the nonsmooth coefficients will introduce new singularities to the solutions so that only limited initial regularity can be propagated.

In [16], Symes proved a trace theorem for the solution of a second order multidimensional wave equation with constant coefficients: For finite energy initial data compactly supported away from the boundary (with the absence of the grazing rays), the trace is of class $H_{l o c}^{1}$ which is as regular as the solution in the interior. Some similar trace regularity results were obtained by Lasiecka and Triggiani [10] for the solutions of second order hyperbolic mixed problems based on the application of the Laplace-Fourier transform. See also Lasiecka and Triggiani [11] for some sharp global trace regularity results for second order hyperbolic equations with smooth coefficients and Neumann boundary conditions. 
Recently in [2], we proved a trace theorem for general linear p.d.e. with smooth variable coefficients, applying the Hörmander-Nirenberg pseudodifferential cutoff technique and the method of energy estimates. Our theorem shows that the difficulty above may be resolved by imposing more smoothness against grazing ray directions.

Roughly speaking, the analysis in this paper is similar to that of Bao and Symes [2]. Two major differences are:

- Since in this work our attention is restricted to the second order equation, compared to the general case in [2] a much simpler $\psi$.d.o. cutoff of the operator becomes possible.

- Note that the model problem has nonsmooth coefficients; therefore the propagation of singularity theorems, Theorems $2.1-2.2$, have to be involved in the analysis in contrast to [2] where the coefficients were assumed to be smooth and no side condition was introduced and discussed explicitly.

In this paper, a trace regularity theorem on a time like surface is proved for the solution of a multidimensional linear acoustic wave equation with nonsmooth coefficients. Our theorem indicates that with microlocal restrictions against tangential oscillations in the coefficient, the boundary value is just as regular as the solution, in particular as regular as the coefficients allow. These properties of traces also indicate that the conclusion of our trace theorem is optimal. However, as compared to the one dimensional problem, a much higher degree of overall smoothness has to be imposed.

Because of the non-hyperbolicity of the operator with respect to a time-like hypersurface, a pseudo-differential cutoff of the operator and results on propagation of singularities are 
necessary in order to apply the method of energy estimates. Moreover, the presence of nonsmooth coefficients demands various results in nonsmooth microlocal analysis.

In Chapter 2, Rauch's lemma on the algebraic property of microlocal Sobolev spaces and a Beals-Reed linear propagation of singularities theorem are extended. Our extension of the Beals-Reed theorem guarantees that under similar hypotheses some lower order microlocal regularity of the solution (for instance, $H_{m l}^{s}$-regularity, for $s \geq 0$ ) will also propagate along the null bicharacteristics.

In Chapter 3, we prove a trace regularity theorem for the solution of a linear acoustic wave equation by using the propagation of singularities theorem introduced in Chapter 2, along with an pseudodifferential ( $\psi$.d.o.) cutoff technique and standard hyperbolic energy estimates.

It is appropriate to conclude this introduction with some general remarks on the usefulness of our methods. In this paper, we only consider a simple second order hyperbolic p.d.e. with nonsmooth coefficients at lower order terms (constant coefficients in the principal part). Without any further difficulty, similar results may also be obtained for higher order hyperbolic p.d.e. with nonsmooth coefficients at lower order terms. A combination of our earlier result in [2] (for smooth variable leading terms) and the ideas in this paper may lead to a analogous trace regularity result for a second order strictly hyperbolic p.d.e. with smooth coefficients at the principal part and nonsmooth coefficients at the lower order terms.

As one can expect, the case when nonsmooth coefficients are present in the principal part of the operator is much more difficult to study. One of the main reasons is because that our Beals-Reed type result on propagation of singularities (Theorem 2.1) is possible because 
the highest order symbol of the operator is smooth, so that a pseudo-differential cut-off could be found following Nirenberg [13] such that the principal part of its commutation with the differential operator vanishes. However, such a pseudo-differential cut-off will not be available when coefficients of the principal part of the operator are nonsmooth, see Beals and Reed [7] for more discussions. For this problem, some partial results have been obtained and will be reported elsewhere.

Notation. Throughout this work, the reader is assumed to be familiar with the basic calculus of Pseudodifferential Operators (" $\psi$.d.o.") as stated in Taylor [18] and Nirenberg [13]. A classical $\psi$.d.o. $P$ of order $m$ is denoted as $P \in O P S^{m}$ with its symbol $p \in S^{m}$. $E S(P)$ stands for the essential support of operator $P$. WF $u$ denotes the wave front set of a distribution $u . H^{s}$ is the standard $L^{2}$-type Sobolev space and $H_{l o c}^{s}$ means a local Sobolev space. $\langle\xi\rangle$ means $\left(1+|\xi|^{2}\right)^{1 / 2}$. The Fourier transform of a distribution $u$ is expressed as $\hat{u}$. Usually, the constant from the Fourier Transform is assumed to be absorbed by the integral. For simplicity, $C$ serves as a generalized positive constant the precise value of which is not needed. Finally, $\chi_{\Gamma}$ is the characteristic function of a set $\Gamma$.

\section{Propagation of Singularities}

Our main result in this chapter is a linear propagation of singularities theorem which is an extension of Theorem 1 in [6] ("the Beals-Reed theorem"). The theorem assures that weaker regularity (than in [6]) of the solution may also be propagated along the null bicharacteristics. The main ingredients in our proof are an extended Rauch's lemma and a commutator lemma. 
We also take care of an interesting special case (which often appears in practice) where the coefficients depend only on some of the variables and show that for this case better regularity results will become possible.

We prove the theorem by following the general scheme of the proof of the Beals-Reed theorem in [6]. The pseudodifferential cutoff technique in their proof was analogous to a proof of Hörmander's theorem (see [8] for the original form) as described in Nirenberg [13]. Except for the use of Rauch's lemma since nonsmooth coefficients and right-hand side were present, the key step was a commutator lemma which allowed them to compute the action on $H^{s} \cap H_{m \ell}^{r}(\gamma)$ of a commutator of a $\psi$.d.o. with a differential operator whose coefficient was nonsmooth. Then a local existence theorem with microlocal hypotheses completed their proof.

The following estimate will be used frequently. Other related kernel estimates may be found in Beals [4] and Beals and Reed [7].

Proposition 2.1 (Rauch and Reed [15]) Define

$$
T_{g, h}(\xi)=\int K(\xi, \eta) f(\eta) g(\xi-\eta) d \eta
$$

where $f, g \in L^{2}$. Then the estimate

$$
\left\|T_{g, h}(\xi)\right\|_{L^{2}} \leq C\|f\|_{L^{2}}\|g\|_{L^{2}}
$$

holds if $K(\xi, \eta)$ can be decomposed into finitely many pieces, i.e. $K=\sum_{i} K_{i}(\xi, \eta)$, each of which satisfies one of the following conditions:

$$
\text { (1) } \sup _{\xi} \int\left|K_{i}(\xi, \eta)\right|^{2} d \eta \leq C_{0}<+\infty
$$


(2) $\sup _{\eta} \int\left|K_{i}(\xi, \eta)\right|^{2} d \xi \leq C_{0}<+\infty$

An immediate consequence of Proposition 2.1 leads to a key estimate in this chapter.

Corollary 2.1 Define

$$
T_{g, h}(\xi)=\int \frac{f(\eta) g(\xi-\eta)}{\langle\eta\rangle^{\alpha}\langle\xi-\eta\rangle^{\beta}} d \eta
$$

where $f, g \in L^{2}\left(\mathbb{R}^{n}\right), \alpha+\beta>n / 2$. Then

$$
\left\|T_{g, h}(\xi)\right\|_{L^{2}} \leq C\|f\|_{L^{2}}\|g\|_{L^{2}}
$$

We will also need:

Proposition 2.2 Assume that $K^{\prime \prime}$ is a closed cone which is strictly contained in an open cone $K$. If $\xi \in K^{\prime \prime}, \eta \in K^{\prime}$, then

(1) $|\xi-\eta| \geq C_{1}|\xi|, C_{1}>0$

(2) if $|\xi| \geq C_{0}>0$, then $\langle\xi-\eta\rangle \geq C\langle\xi\rangle$.

\subsection{Microlocal Sobolev spaces}

We present some basic properties of microlocal Sobolev spaces. Only new results will be proved.

The standard Schauder's lemma asserts that $H^{s}\left(\mathbb{R}^{n}\right)$ is an algebra for $s>n / 2$. Concerning the lower order Sobolev spaces, one can generalize Schauder's lemma in a number of ways.

Lemma 2.1 If $u \in H^{s_{1}}\left(\mathbb{R}^{n}\right)$ and $v \in H^{s_{2}}\left(\mathbb{R}^{n}\right)$, with $s_{1}+s_{2} \geq 0$, then

$$
u v \in H^{\min \left(s_{1}, s_{2}, s_{1}+s_{2}-n / 2-\delta\right)} \quad \text { for any } \delta>0
$$


See Beals [4] for a proof when either $s_{1}, s_{2} \geq 0$ or $s_{1}, s_{2} \leq n / 2$ and $s_{1}+s_{2} \geq 0$. The complete proof may be found in Bao [1].

For family of distributions, we state a slightly weaker algebraic property.

Lemma 2.2 (Generalized Schauder's Lemma) If $s_{1}, s_{2} \geq 0, u(x) \in H^{s_{1}}\left(\mathbb{R}^{n_{0}}\right), v(x, y) \in$ $H^{s_{2}}\left(\mathbb{R}^{n}\right)$, and $1 \leq n_{0} \leq n$. Then

$$
u(x) v(x, y) \in H^{s}\left(\mathbb{R}^{n}\right)
$$

with $s=\min \left\{s_{1}, s_{2}, s_{1}+s_{2}-n_{0} / 2-\delta\right\}$, for any $\delta>0$.

The proof is a combination of some kernel estimates and Young's inequality, see [1] for a complete proof.

It is evident that the microlocal Sobolev spaces give a precise description about how regularity and singularities are propagated for solutions to linear strictly hyperbolic p.d.e..

Definition $2.1 u \in H^{s} \cap H_{m \ell}^{r}\left(x_{0}, \xi_{0}\right)$ if there exist $\phi(x) \in C_{0}^{\infty}\left(\mathbb{R}^{n}\right)$ with $\phi\left(x_{0}\right) \neq 0$ and a conic neighborhood $\gamma \subset \mathbb{R}^{n} \backslash\{0\}$ of $\xi_{0}$ such that

$$
\langle\xi\rangle^{s}(\phi u)^{\wedge}(\xi) \in L^{2}\left(\mathbb{R}^{n}\right) \text { and }\langle\xi\rangle^{r} \chi_{\gamma}(\xi)(\phi u)^{\wedge}(\xi) \in L^{2}\left(\mathbb{R}^{n}\right)
$$

To work on microlocal Sobolev spaces, Rauch's lemma is essential. It gives the algebraic property of this interesting class of spaces. Here, we prove an extended Rauch's lemma.

Lemma 2.3 Suppose that for some $\left(x_{0}, \xi_{0}\right) \in T^{*}\left(\mathbb{R}^{n_{0}}\right) \backslash 0$ where $\left(x_{0}, y_{0}, \xi_{0}, \eta_{0}\right) \in T^{*}\left(\mathbb{R}^{n}\right) \backslash 0$ $\left(1 \leq n_{0} \leq n\right)$, the distributions $u$, v satisfy $u(x) \in H^{s} \cap H_{m \ell}^{q}\left(x_{0}, \xi_{0}\right)$ and $v(x, y) \in H^{l} \cap$ $H_{m \ell}^{q}\left(x_{0}, y_{0}, \xi_{0}, \eta_{0}\right)$, with $n_{0} / 2<s, 0 \leq l \leq s, q$, and $q<l+s-n_{0} / 2$. Then

$$
u(x) v(x, y) \in H^{l} \cap H_{m \ell}^{q}\left(x_{0}, y_{0}, \xi_{0}, \eta_{0}\right)
$$


Proof. The fact that $u v \in H_{l o c}^{l}$ comes from Lemma 2.2. W.L.O.G., we may assume that $u, v$ have compact supports in their own spaces. Moreover, we only prove the lemma for the case $q>s$; a natural modification of the proof will yield the conclusion for $q \leq s$. Let $K$ be a conic neighborhood of $\left(\xi_{0}, \eta_{0}\right)$ which is small enough so that $v \in H^{l} \cap H_{m \ell}^{q}(K)$ and $u \in H^{s} \cap H_{m \ell}^{q}(\Gamma)$, where $\Gamma$ is the projection of $K$ on the $\xi$-space. Let $K^{\prime} \subset \subset K$, a strictly smaller conic neighborhood of $\left(\xi_{0}, \eta_{0}\right) . \Gamma^{\prime}$ is the projection of $K^{\prime}$. It suffices to show that

$$
\widehat{u v}(\xi, \eta) \chi_{K^{\prime}}(\xi, \eta)\langle\xi, \eta\rangle^{q} \in L^{2}\left(\mathbb{R}^{n}\right)
$$

Write $u=u_{1}+u_{2}, v=v_{1}+v_{2}$ such that

$$
\begin{aligned}
& u_{1} \in H^{s}, \quad u_{2} \in H^{q}, \text { and } \operatorname{supp} \hat{u}_{1} \subseteq \Gamma^{c}, \text { supp } \hat{u}_{2} \subseteq \Gamma, \\
& v_{1} \in H^{l}, \quad v_{2} \in H^{q}, \text { and supp } \hat{v}_{1} \subseteq K^{c}, \text { supp } \hat{v}_{2} \subseteq K
\end{aligned}
$$

Then

$$
u v=u_{1} v_{1}+u_{1} v_{2}+u_{2} v_{1}+u_{2} v_{2} .
$$

According to Lemma $2.2, u_{2} v_{2} \in H^{q}$, therefore it suffices to show that

$$
W=u v-u_{2} v_{2}=u_{1} v_{1}+u_{1} v_{2}+u_{2} v_{1} \in H^{l} \cap H_{m \ell}^{q}\left(K^{\prime}\right) .
$$

Let

$$
\begin{array}{ll}
\langle\xi\rangle^{s} \hat{u}_{1}(\xi)=f_{1}(\xi), & \langle\xi\rangle^{q} \hat{u}_{2}(\xi)=f_{2}(\xi), \\
\langle\xi, \eta\rangle^{l} \hat{v}_{1}(\xi, \eta)=g_{1}(\xi, \eta), & \langle\xi, \eta\rangle^{q} \hat{v}_{2}(\xi, \eta)=g_{2}(\xi, \eta),
\end{array}
$$

then $f_{i} \in L^{2}\left(\mathbb{R}^{n_{0}}\right)$ and $g_{i} \in L^{2}\left(\mathbb{R}^{n}\right), i=1,2$. Thus we may decompose

$$
\langle\xi, \eta\rangle^{q} \Upsilon_{K^{\prime}}(\xi, \eta) \widehat{W}(\xi, \eta)=I_{1}+I_{2}+I_{3}
$$


where

$$
\begin{aligned}
& I_{1}=\langle\xi, \eta\rangle^{q} \int \frac{\chi_{K^{\prime}}(\xi, \eta) \chi_{\Gamma}\left(\xi_{1}\right) \chi_{K^{c}}\left(\xi-\xi_{1}, \eta\right) f_{2}\left(\xi_{1}\right) g_{1}\left(\xi-\xi_{1}, \eta\right) d \xi_{1}}{\left\langle\xi_{1}\right\rangle^{q}\left\langle\xi-\xi_{1}, \eta\right\rangle^{l}} \\
& I_{2}=\langle\xi, \eta\rangle^{q} \int \frac{\chi_{K^{\prime}}(\xi, \eta) \chi_{\Gamma^{c}}\left(\xi_{1}\right) \chi_{K^{k}}\left(\xi-\xi_{1}, \eta\right) f_{1}\left(\xi_{1}\right) g_{2}\left(\xi-\xi_{1}, \eta\right) d \xi_{1}}{\left\langle\xi_{1}\right\rangle^{s}\left\langle\xi-\xi_{1}, \eta\right\rangle^{q}} \\
& I_{3}=\langle\xi, \eta\rangle^{q} \int \frac{\chi_{K^{\prime}}(\xi, \eta) \chi_{\Gamma^{c}}\left(\xi_{1}\right) \chi_{K^{c}}\left(\xi-\xi_{1}, \eta\right) f_{1}\left(\xi_{1}\right) g_{1}\left(\xi-\xi_{1}, \eta\right) d \xi_{1}}{\left\langle\xi_{1}\right\rangle^{s}\left\langle\xi-\xi_{1}, \eta\right\rangle^{l}}
\end{aligned}
$$

Therefore, to accomplish the proof, we only need to show that $I_{i} \in L^{2}\left(\mathbb{R}^{n}\right)(i=1, \cdots, 3)$.

On support $I_{1},\left(\xi-\xi_{1}, \eta\right) \in K^{c}$ and $(\xi, \eta) \in K^{\prime} \Rightarrow\left\langle\xi_{1}, 0\right\rangle \geq C\langle\xi, \eta\rangle$. Hence

$$
\left|I_{1}\right| \leq \int \frac{\left|f_{2}\left(\xi_{1}\right) g_{1}\left(\xi-\xi_{1}, \eta\right)\right| d \xi_{1}}{\left\langle\xi-\xi_{1}, \eta\right\rangle^{l}}
$$

therefore an extension of Corollary 2.1 and the hypotheses will yield

$$
\left\|I_{1}\right\|_{L^{2}\left(\mathbb{R}^{n}\right)} \leq C\left\|g_{1}\right\|_{L^{2}}\left\|f_{2}\right\|_{L^{2}}
$$

On support $I_{2}, \xi_{1} \in \Gamma^{c},(\xi, \eta) \in K^{\prime} \Rightarrow\left\langle\xi-\xi_{1}, \eta\right\rangle \geq C\langle\xi, \eta\rangle$, Young's inequality gives

$$
\left\|I_{2}\right\|_{L^{2}(\xi)} \leq C\left\|\frac{\left|f_{1}(\xi)\right|}{\langle\xi\rangle^{s}}\right\|_{L^{1}(\xi)}\left\|g_{2}\right\|_{L^{2}\left(\mathbb{R}^{n}\right)}
$$

Thus, one gets from the hypothesis $s>n_{0} / 2$ that

$$
\left\|I_{2}\right\|_{L^{2}\left(\mathbb{R}^{n}\right)} \leq C\left\|f_{1}\right\|_{L^{2}\left(\mathbb{R}^{n_{0}}\right)}\left\|g_{2}\right\|_{L^{2}\left(\mathbb{R}^{n}\right)}
$$

On support $I_{3}, \xi_{1} \in \Gamma^{c},\left(\xi-\xi_{1}, \eta\right) \in K^{c},(\xi, \eta) \in K$. Then, since $q<l+s-n_{0} / 2$, one may apply an extension form of Proposition 2.3 below to obtain

$$
\left\|I_{3}\right\|_{L^{2}\left(\mathbb{R}^{n}\right)} \leq C\left\|f_{1}\right\|_{L^{2}}\left\|g_{1}\right\|_{L^{2}}
$$


Remark on Lemma 2.3. Note that for $n_{0}=n$ and $l=s$, this lemma becomes the original Rauch's lemma. Since $l$ can be any constant between 0 and $s, q$ can be any number in the interval $\left[l, s+l-n_{0} / 2\right.$ ), Lemma 2.3 is indeed an improvement of Rauch's lemma (even when $\left.n_{0}=n\right)$.

We claim that like Rauch's lemma, Lemma 2.3 cannot be strengthened. The following result and an example exhibit the extent to which the result is applicable.

Proposition 2.3 Let $K_{1}, K_{2}$, and $K$ be cones in $\mathbb{R}^{n} \backslash 0$ and assume that $u_{i} \in H^{s_{i}}\left(\mathbb{R}^{n}\right)$ and $\tilde{\Pi} W F\left(w_{i}\right) \subset K_{i}, i=1,2$, where $\grave{\Pi}$ denotes the projection on the second factor (or on the frequency space). If $K \subset \subset K_{1}^{c} \cap K_{2}^{c}$, then

$$
\chi_{K}(D)\left(w_{1} w_{2}\right) \in H^{\tilde{s}}, \text { if } \tilde{s} \leq s_{1}+s_{2}-n / 2 .
$$

Remark. When $\tilde{s}<s_{1}+s_{2}-n / 2$, the result is due to Rauch (Theorem 2.2 in [14]); the extreme case was first observed by Meyer in [12]. The proposition cannot be strengthened because an example of Beals, see Beals [5] pages 15-16 for details.

\subsection{Commutator lemma}

Having introduced the basic concepts of microlocal Sobolev spaces, we now present a commutator lemma which is necessary in order to prove any results on propagation of singularities for a p.d.e. with nonsmooth coefficients.

Lemma 2.4 (Generalized Commutator Lemma) Let $p_{1}(x, y, \xi, \eta) \in S^{1}\left(\mathbb{R}^{n}\right)$ and $b_{0}(x, y, \xi, \eta) \in$ $S^{0}\left(\mathbb{R}^{n}\right)$, and assume that for some $\left(x_{0}, \xi_{0}\right) \in T^{*}\left(\mathbb{R}^{n_{0}}\right),\left(x_{0}, y_{0}, \xi_{0}, \eta_{0}\right) \in T^{*}\left(\mathbb{R}^{n}\right)\left(1 \leq n_{0} \leq\right.$ 
n), $a(x) \in H^{s} \cap H_{m \ell}^{q}\left(x_{0}, \xi_{0}\right)$ and $v(x, y) \in H^{l} \cap H_{m \ell}^{q}\left(x_{0}, y_{0}, \xi_{0}, \eta_{0}\right)$, with $1+n_{0} / 2<s, 0 \leq l \leq s$, $q$, and $q<l+s-\left(1+n_{0} / 2\right)$. Then

$$
\left[b_{0}, a p_{1}\right] v \in H^{l} \cap H_{m \ell}^{q}\left(x_{0}, y_{0}, \xi_{0}, \eta_{0}\right)
$$

We shall only prove this lemma for the case $n_{0}=n$; the general case follows after some obvious modifications. The proof contains two steps: Proposition 2.4 offers the local version of the commutator action, while the microlocal version is given as Proposition 2.5.

Proposition 2.4 Let $p_{1}(x, \xi) \in S^{1}, b_{\mathrm{u}}(x, \xi) \in S^{0}$ be properly supported, let $1+n / 2<s$, and assume that $a(x) \in H^{s}$ and $v(x) \in H^{l}$ with $0 \leq l \leq s$. Then

$$
\left[b_{0}(x, D), a(x) p_{1}(x, D)\right] v(x) \in H^{l}
$$

Proof. Assume that $b_{0}, p_{1}$ depend on $\xi$ only, and $v, a$ are compactly supported (the general case requires some obvious modifications). We only consider $l \geq 1$ case. A slightly different analysis will lead to the conclusion for $0 \leq l<1$.

$$
\begin{aligned}
& \overbrace{\left[b_{0}(D), a(x) p_{1}(D)\right] v}(\xi) \\
& \quad=b_{0}(\xi) \int \hat{a}(\eta) p_{1}(\xi-\eta) \hat{v}(\xi-\eta) d \eta-\int \hat{a}(\eta) p_{1}(\xi-\eta) b_{0}(\xi-\eta) \hat{v}(\xi-\eta) d \eta \\
& \quad=\int \hat{a}(\eta)\left(b_{0}(\xi)-b_{0}(\xi-\eta)\right) p_{1}(\xi-\eta) \hat{v}(\xi-\eta) d \eta .
\end{aligned}
$$

Write $\hat{a}(\eta)=f(\eta) /\langle\eta\rangle^{s}, \hat{v}(\xi-\eta)=g(\xi-\eta) /\langle\xi-\eta\rangle^{l}$, then $f, g \in L^{2}$. Thus

$$
\langle\xi\rangle^{l} \overbrace{\left[b_{0}(D), a(x) p_{1}(D)\right] v}(\xi)=\int K(\xi, \eta) f(\eta) g(\xi-\eta) d \eta
$$

where

$$
K(\xi, \eta) \leq \frac{C\langle\xi\rangle^{l}\left|b_{0}(\xi)-b_{0}(\xi-\eta)\right|}{\langle\eta\rangle^{s}\langle\xi-\eta\rangle^{l-1}}
$$


By Proposition 2.1, it suffices to divide $K$ into finitely many pieces so that

$$
\sup _{\xi} \int\left|K_{i}(\xi, \eta)\right|^{2} d \eta<\infty, \quad \text { or } \sup _{\eta} \int\left|K_{i}(\xi, \eta)\right|^{2} d \xi<\infty
$$

But this is obvious from the given regularity assumptions and Proposition 2.2, together with the following facts: For $|\eta|>|\xi| / 2$ and $|\xi-\eta|>|\xi| / 2$,

$$
|K| \leq \frac{C}{\langle\xi\rangle^{s-1}}
$$

For $|\eta|>|\xi| / 2$ and $|\xi-\eta|<|\xi| / 2$,

$$
|K| \leq \frac{C\langle\xi\rangle^{l}}{\langle\xi\rangle^{s}\langle\xi-\eta\rangle^{l-1}} \leq \frac{C}{\langle\xi-\eta\rangle^{s-1}}
$$

For $|\eta|<|\xi| / 2, \xi-\eta \approx \xi$, thus $\left|b_{0}(\xi)-b_{0}(\xi-\eta)\right| \leq C\langle\eta\rangle /\langle\xi\rangle$, and consequently,

$$
|K| \leq \frac{C\langle\xi\rangle^{l}\langle\eta\rangle}{\langle\eta\rangle^{s}\langle\xi\rangle^{l-1}\langle\xi\rangle}=\frac{C}{\langle\eta\rangle^{s-1}}
$$

Proposition 2.5 Let $p_{1}(x, \xi) \in S^{1}, b_{0}(x, \xi) \in S^{0}$ be properly supported, and assume that for some $\left(x_{0}, \xi_{0}\right) \in T^{*}\left(\mathbb{R}^{n}\right) \backslash 0, a(x) \in H^{s} \cap H_{m \ell}^{q}\left(x_{0}, \xi_{0}\right)$ and $v(x) \in H^{l} \cap H_{m \ell}^{q}\left(x_{0}, \xi_{0}\right)$, with $1+n / 2<s, 0 \leq l \leq s, q$ and $q<l+s-(1+n / 2)$. Then

$$
\left[b_{0}(x, D), a(x) p_{1}(x, D)\right] v(x) \in H_{m \ell}^{q}\left(x_{0}, \xi_{0}\right) .
$$

Proof. As before, after maling some simplifications, we have

$$
\overbrace{\left[b_{0}(D), a(x) p_{1}(D)\right] v}(\xi)=\int \hat{a}(\eta)\left(b_{0}(\xi)-b_{0}(\xi-\eta)\right) p_{1}(\xi-\eta) \hat{v}(\xi-\eta) d \eta .
$$


Let $K$ be a small conic neighborhood of $\xi_{0}$ such that $a \in H^{s} \cap H_{m \ell}^{q}(K)$ and $v \in H^{l} \cap H_{m \ell}^{q}(K)$. Let $K^{\prime}$ be a strictly smaller conic neighborhood of $\xi_{0}$; then in order to prove (2.1) it suffices to show that

$$
\chi_{K^{\prime}}(\xi)\langle\xi\rangle^{q} \overbrace{\left[b_{0}(D), a(x) p_{1}(D)\right] v}(\xi) \in L^{2}
$$

Write

$$
\begin{aligned}
\hat{a}(\eta) & =\frac{\chi_{K}(\eta) a_{1}(\eta)}{\langle\eta\rangle^{r}}+\frac{\chi_{K^{c}}(\eta) a_{2}(\eta)}{\langle\eta\rangle^{s}} \\
\hat{v}\langle\xi-\eta\rangle & =\frac{\chi_{K}(\xi-\eta) v_{1}(\xi-\eta)}{\langle\xi-\eta\rangle^{q}}+\frac{\chi_{K^{c}}(\xi-\eta) v_{2}(\xi-\eta)}{\langle\xi-\eta\rangle^{l}}
\end{aligned}
$$

where $a_{i}, v_{i} \in L^{2}(i=1,2)$.

We only prove (2.1) for the case where $q>s$, since if $q \leq s$ then (2.3) will become $\hat{a}(\eta)=\frac{a_{1}(\eta)}{\langle\eta\rangle^{s}}$, and the same analysis will go through with much simpler arguments.

Substituting (2.3) and (2.4) into (2.2), we then have

$$
\chi_{K^{\prime}}\langle\xi\rangle^{q} \overbrace{\left[b_{0}, a P_{1}\right] v}(\xi)=\sum_{i, j=1}^{2} \int K_{i j}(\xi, \eta) a_{i}(\xi) v_{j}(\xi-\eta) d \eta
$$

where

(1) $K_{11}(\xi, \eta)=\frac{\chi_{K^{\prime}}(\xi)\langle\xi\rangle^{q} \chi_{K^{*}}(\eta) \chi_{K^{*}}(\xi-\eta)\left(b_{0}(\xi)-b_{0}(\xi-\eta)\right)\langle\xi-\eta\rangle}{\langle\eta\rangle^{q}\langle\xi-\eta\rangle^{q}}$

(2) $K_{12}(\xi, \eta)=\frac{\chi_{K^{\prime}}(\xi)\langle\xi\rangle^{q} \chi_{K^{*}}(\eta) \chi_{K^{c}}(\xi-\eta)\left(b_{0}(\xi)-b_{0}(\xi-\eta)\right)\langle\xi-\eta\rangle}{\langle\eta\rangle^{q}\langle\xi-\eta\rangle^{l}}$,

(3) $K_{21}(\xi, \eta)=\frac{\chi_{K^{\prime}}(\xi)\langle\xi\rangle^{q} \chi_{K^{c}}(\eta) \chi_{K}(\xi-\eta)\left(b_{0}(\xi)-b_{0}(\xi-\eta)\right)\langle\xi-\eta\rangle}{\langle\eta\rangle^{s}\langle\xi-\eta\rangle^{q}}$,

(4) $K_{22}(\xi, \eta)=\frac{\chi K^{\prime}(\xi)\langle\xi\rangle^{q} \chi K^{c}(\eta) \chi_{K^{c}}(\xi-\eta)\left(b_{0}(\xi)-b_{0}(\xi-\eta)\right)\langle\xi-\eta\rangle}{\langle\eta\rangle^{s}\langle\xi-\eta\rangle^{l}}$.

It follows from Proposition 2.1 (essentially Cauchy-Schwarz inequality) that the corresponding estimates of the kernels will complete the proof: 
(1) $K_{11}$ will be handled exactly as in Proposition 2.4 , knowing the fact that $1+n / 2<q$.

(2) Since $q>s$, the hypothesis implies that $l-1>n / 2$. On supp $K_{12}, \xi-\eta \in K^{c}$, $\xi \in K^{\prime \prime} \Rightarrow\langle\eta\rangle \geq C\langle\xi\rangle ;$ hence

$$
\begin{aligned}
& \text { if }|\xi-\eta| \geq C|\xi|, \quad \text { then }\left|K_{12}\right| \leq \frac{C}{\langle\xi\rangle^{l-1}}, \\
& \text { if }|\xi| \geq C|\xi-\eta|, \text { then }\left|K_{12}\right| \leq \frac{C}{\langle\xi-\eta\rangle^{l-1}} .
\end{aligned}
$$

(3) On supp $K_{21}, \eta \in K^{c}, \xi \in K^{\prime} \Rightarrow\langle\xi-\eta\rangle \geq C\langle\xi\rangle$. Now

$$
\begin{aligned}
& \text { if }|\eta| \geq|\xi| / 2, \text { then }\left|K_{21}\right| \leq \frac{C}{\langle\xi-\eta\rangle^{s-1}}, s-1>n / 2 ; \\
& \text { if }|\eta|<|\xi| / 2 \text { then } \xi-\eta \approx \xi \text {, thus }\left|b_{0}(\xi)-b_{0}(\xi-\eta)\right| \leq C\langle\eta\rangle /\langle\xi\rangle,
\end{aligned}
$$

and it follows that $K_{21} \leq \frac{C\langle\xi\rangle^{q}\langle\eta\rangle\langle\xi-\eta\rangle}{\langle\eta\rangle^{s}\langle\xi-\eta\rangle^{q}\langle\xi\rangle} \leq \frac{C}{\langle\eta\rangle^{s-1}}$ for $s-1>n / 2$.

(4) On supp $K_{22}, \eta \in K^{c}, \xi-\eta \in K^{c}, \xi \in K^{\prime \prime} \Rightarrow$ Proposition 2.3 can be applied to treat this term since $q \leq l+s-(1+n / 2)$.

\subsection{Propagation of singularities theorem}

We are now ready for a formal statement of the main result of this chapter: a linear propagation of singularities theorem for $\psi$.d.o. equations with nonsmooth coefficients at lower order terms. The proof follows exactly the proof of Beals-Reed theorem in [6] with the original Rauch's lemma replaced by its extension Lemma 2.3 and the commutator lemma of [6] replaced by Lemma 2.4. Therefore we omit the proof. 
Theorem 2.1 Let $p_{m}(x, D)$ be a strictly hyperbolic homogeneous $\psi$.d.o. of degree $m \geq 2$, $p_{\alpha}(x, \xi) \in S^{m-1}, p_{\beta}(x, \xi) \in S^{m-2}$, with $x=\left(x_{1}, x_{2}\right) \in \mathbb{R}^{n_{0}} \times \mathbb{R}^{n-n_{0}}, 1 \leq n_{0} \leq n$. Let $\Gamma$ be a null bicharacteristic of $p_{m}$ passing through $\left(x_{0}, \xi_{0}\right) \in T^{*}\left(\mathbb{R}^{n}\right) \backslash 0$. Denote $K=\Pi(\Gamma)$, $\Pi: T^{*}\left(\mathbb{R}^{n}\right) \rightarrow T^{*}\left(\mathbb{R}^{n_{0}}\right)$ the projection map. Assume that

$$
\begin{aligned}
& \text { (i) } 1+n_{0} / 2<s, 0 \leq l \leq s, q \text {, and } q<l+s-\left(1+n_{0} / 2\right) \text {; } \\
& \text { (ii) } a_{\alpha} \in H^{s} \cap H_{m \ell}^{q}(K) \text { and } a_{\beta} \in H^{s-1} \cap H_{m \ell}^{q-1}(K) ; \\
& \text { (iii) } v \in H^{l+m-2} \cap H_{m \ell}^{q+m-2}(\Gamma) \text { and } f \in H^{l} \cap H_{m \ell}^{q}(\Gamma) \text {; } \\
& \text { (iv) } v \in H_{m \ell}^{q+m-2+\varepsilon}\left(x_{0}, \xi_{0}\right) \text {, for some } 0 \leq \epsilon \leq 1,
\end{aligned}
$$

and that

$$
\left[p_{m}(x, D)+\Sigma a_{\alpha}\left(x_{1}\right) p_{\alpha}(x, D)+\Sigma a_{\beta}\left(x_{1}\right) p_{\beta}(x, D)\right] v(x)=f(x)
$$

Then

$$
v \in H_{m \ell}^{q+m-2+\epsilon}(\Gamma)
$$

An immediate consequence of Theorem 2.2 is a theorem on propagation of singularities due to Beals and Reed, Theorem 1 in [6].

Remark on Theorem 2.1.

Notice that the Beals-Reed theorem as well as Rauch's Lemma are designed for the study of nonlinear propagation of singularities. In that case the coefficients or the right-hand side, roughly speaking, have same (or closely related) regularity as the solution to the problem. Theorem 2.1 deals with much more general situations, since $l$ could vary from 0 to $s$ and $q \in\left[l, l+s-\left(1+n_{0} / 2\right)\right)$ (unlike in the Beals-Reed theorem). 
We conjecture that Theorem 2.1 cannot be improved much concerning the regularity requirements for the coefficients and right-hand side, since the conclusions of Lemma 2.3 and Lemma 2.4 cannot be strengthened.

The most precise information about the propagation of singularities may be obtained in the case of one space dimension. Roughly speaking, the improved microlocal regularity is then propagated along null bicharacteristics with very few restriction on the order of smoothness. This certainly is not implied by Theorem 2.1. Note that the result itself is not too surprising if one observes that the one dimensional wave operator can be factored into products of differential operators. But it suggests a substantial difference between the one dimension and multidimension for hyperbolic p.d.e., which is somehow remarkable.

\section{Trace Regularity Theorem}

\subsection{Properties of $\psi$.d.o.-like operators}

It is evident that a smooth family of 4.d.o. $P\left(x, y, D_{x}\right) \in O P S^{m}\left(\mathbb{R}^{k_{0}}\right)$, for each $y \in \mathbb{R}^{k-k_{0}}$ with $k_{0}<k$, which may be denoted as $P \in C^{\infty}\left(\mathbb{R}^{k-k_{0}}, O P S^{m}\left(\mathbb{R}^{k_{0}}\right)\right)$, is not necessarily a $\psi$.d.o. in $\mathbb{R}^{k}$, see Bao and Symes [2] or Taylor [17]. In [2], we concluded that a smooth family of $\psi$.d.o. in fact behaves like a $\psi . d . o$, , hence will be called a $\psi$.d.o.-like operator.

From now on, $\tilde{\Pi}_{2}: X \in T^{*}\left(\mathbb{R}^{k}\right) \rightarrow Y \in \mathbb{R}^{k} \times \mathbb{R}^{k_{0}}$ serves as a map for $k>k_{0}$,

$$
\hat{\Pi}_{2}(X)=\{(x, y, \xi) \in Y:(x, y, \xi, \eta) \in X\}
$$


Recall that the normal bundle of a foliation $\mathbb{R}^{k}=\mathbb{R}^{k-k_{0}} \times \mathbb{R}^{k_{0}}$ is the set

$$
\mathcal{N}=\left\{(x, y, \xi, \eta) \in \mathbb{R}^{k_{0}} \times \mathbb{R}^{k-k_{0}} \times \mathbb{R}^{k_{0}} \times \mathbb{R}^{k-k_{0}}, \xi=0\right\}
$$

We now recall a fattening lemma (the Lemma in Bao and Symes [2]). The usefulness of this lemma will become clear in the proofs of the coming trace theorems.

Lemma 3.1 (Fattening Lemma) Let $B\left(x, y, D_{x}\right) \in C^{\infty}\left(\mathbb{R}^{k-k_{0}}, O P S^{m}\left(\mathbb{R}^{k_{0}}\right)\right.$ and $A\left(x, y, D_{x}, D_{y}\right) \in$ $\operatorname{OPS} \mathrm{m}_{0}\left(\mathbb{R}^{k}\right)$, where $1 \leq k_{0} \leq k$. Let

$$
\mathcal{N}=\left\{(x, \xi) \in \mathbb{R}^{k} \times \mathbb{R}^{k},\left(\xi_{1}, \cdots, \xi_{k_{0}}\right)=0\right\}
$$

be the normal bundle of $\mathbb{R}^{k_{0}} \times \mathbb{R}^{k-k_{0}}$. Also, assume that

(1) $A$ is microlocal elliptic on a conic set Ell $(A)$, with $\mathcal{N} \subset \subset \operatorname{Ell}(A)$;

(Q) $u \in H^{h} \cap H_{m \ell}^{h+1}\left(\left[T^{*}\left(\mathbb{R}^{k}\right) \backslash \operatorname{Ell}(A)\right] \cap \tilde{\Pi}_{2}^{-1} \operatorname{ES}(B(\cdot, y, \cdot))\right)$;

(3) A $\phi u \in H_{\text {loc }}^{h-m_{0}+1}\left(\mathbb{R}^{k}\right)$, where $\phi(x) \in C_{0}^{\infty}\left(\mathbb{R}^{k}\right)$.

Then

$$
B \phi u \in H_{\mathrm{loc}}^{h-m+1}\left(\mathbb{R}^{k}\right)
$$

in addition, if $B$ is either a convolutional operator or its symbol has compact support in spatial variables,

$$
B \phi u \in H^{h-m+1}\left(\mathbb{R}^{k}\right)
$$

Remark. The lemma provides a convenient way to determine the regularity of the action of a smooth family of $\psi$.d.o. on a distribution under some appropriate hypotheses. This lemma plays a dominant role in deriving our trace regularity theorem. 
Furthermore, an estimate can be obtained by carrying out all the corresponding estimates in the proof of Lemma 3.1:

Lemma 3.1' Under the assumptions of Lemma 3.1, the following estimate holds:

$$
\left\|\phi_{0} B \phi u\right\|_{h-m+1} \leq C\|\phi u\|_{h}+C\left\|\phi_{0} A \phi u\right\|_{h-m_{0}+1}+C\left\|\phi_{0} P \phi u\right\|_{h+1}
$$

where $\phi_{0}(x) \in C_{0}^{\infty}\left(\mathbb{R}^{k}\right), P \in O P S^{0}$ and $E S(P) \subseteq$ a sufficiently small conic neighborhood of $\Gamma=\left[T^{*}\left(\mathbb{R}^{k}\right) \backslash E l_{1}\right] \cap \tilde{\Pi}_{2}^{-1} E S(B(\cdot, y, \cdot))$ and $p=1$ on $\Gamma \cap\{(x, \xi):|\xi|>1\}$.

\subsection{Construction of a cutoff}

From now on, the space variable is always denoted as $\left(x^{\prime}, x_{n}\right) \in \mathbb{R}^{n-1} \times \mathbb{R}, n \geq 2$, and the Fourier variables dual to $t, x$ are $\omega, \xi$ respectively.

For convenience, we construct a cutoff in Lemma 3.2 below by following Nirenberg's construction of $\psi$.l.o. cutoffs in [13]. Although the lemma is stated for the wave operator $\square=\partial_{t}^{2}-\Delta(\Delta$ is the $n$-dimensional Laplacian), which is what we need in this work, it is clear from the proof that the corresponding result for general operators can be established with no further difficulty.

Let

$$
\square(x, t, \xi, \omega)=(1 / 2)\left(\omega^{2}-|\xi|^{2}\right),
$$

the bicharacteristic strips of $\square$ are defined by the Hamiltonian System

$$
\begin{array}{ll}
\dot{x}=-\xi, & \dot{\xi}=0, \\
\dot{t}=\omega, & \dot{\omega}=0 .
\end{array}
$$


The null bicharacteristics of $\square$ are those that satisfy $\omega^{2}=|\xi|^{2}$. For example, one can easily write down the characteristic through the point $\left(x_{0}, 0, \xi_{0}, \omega_{0}\right)$ with $\omega_{0}^{2}=\left|\xi_{0}\right|^{2}$ as

$$
\left\{(x, t, \xi, \omega): x=x_{0}-\left(\xi_{0} / \omega_{0}\right) t, \xi=\xi_{0} \text {, and } \omega=\omega_{0}\right\} \text {. }
$$

Lemma 3.2 Given a conic set $\gamma$, there exists a $B \in O P S^{0}$ such that

(1) $[B, \square] \in O P S^{\circ}$;

(2) $B$ is elliptic on the null bicharacteristics (Hamiltonian flow) generated by the wave operator $\square$ out of $\gamma$.

Proof. According to Nirenberg's proof of the theorem of propagation of singularities, we can find a $\psi$.d.o. $A$ of order zero for every null bicharacteristic of $\square$ out of $\gamma$ such that $A$ is elliptic on a small conic neighborhood of the bicharacteristic and $[A, \square] \in O P S^{0}$.

Now $B$ may be constructed in the following way: $B=\sum A$ where $A$ is defined as above. Then $B \in O P S^{0}$, it can be arranged to be elliptic on the Hamiltonian flow out of $\gamma$, and $[B, \square] \in O P S^{0}$. Moreover, the local compactness of the unit sphere ensures that the summation is finite.

Remark: The same idea could lead to the existence of $\tilde{B} \in O P S^{0}$ with all the properties of $B$ and, moreover, $[\tilde{B}, \square] \in O P S^{-\infty}$. However, it is evident that with the presence of nonsmooth coefficients, the fact that $[\dot{B}, \square] \in O P S^{-\infty}$ will not benefit our analysis any further. 


\subsection{Trace theorem}

In this section, we present a trace theorem for a linear acoustic wave equation with nonsmooth coefficients at lower order terms. With the presence of nonsmooth coefficients, the extended Beals-Reed theorem (Theorem 2.1) is necessary to describe how the singularities are propagated.

In order to derive the corresponding trace estimate on the solution, we need a Gårding's type inequality concerning the microlocal ellipticity. A simple exercise of the calculus of $\psi$.d.o. gives the proof, see Bao [1] for details.

Lemma 3.3 Assume that $Q_{1} \in O P S^{m_{1}}, Q_{2} \in O P S^{m_{2}}$, with $m_{1}, m_{2} \in \mathbb{R}$. Furthermore assume $Q_{2}$ is elliptic on $E S\left(Q_{1}\right)$. Then for any $r \in \mathbb{R}, \Omega$ and $\Omega^{\prime}$ two open bounded sets of $\mathbb{R}^{n}$ with $\Omega \subset \subset \Omega^{\prime}$, and $u \in C_{0}^{\infty}(\Omega)$.

$$
\left\|Q_{1} u\right\|_{s, \Omega} \leq C\left\|Q_{2} u\right\|_{s+m_{1}-n_{2}, \Omega^{\prime}}+C\|u\|_{r, \Omega^{\prime}}
$$

It is convenient to introduce a useful notation.

Definition 3.1 Let $\Omega \subset \mathbb{R}^{n}$ be open and bounded, $\gamma \subset T^{*}(\Omega)$. A constant $C$ is said to depend on the $H^{s} \cap H_{m e}^{r}(\gamma)$-norm of $u \in C_{0}^{\infty}\left(\mathbb{R}^{k}\right)$ if for any conic neighborhood $\Gamma$ of $\gamma$ there exists a $\psi . d . o$. of order zero with

- $E S(Q) \subseteq \Gamma$

- $q=1$ on $\gamma \cap\{(x, \xi):|\xi|>1\}$

and $C$ depends on $\|u\|_{s}+\|Q u\|_{r, \Omega}$. 
We are now ready to show a trace regularity theorem.

Theorem 3.1 Suppose that $s>3+n / 2$ and that $u$ solves the problem

$$
\begin{gathered}
{[\square-\nabla \sigma(x) \cdot \nabla] u(x, t)=f(x, t),} \\
u(x, t) \in H^{l} \cap H_{m \ell}^{l+1}(\gamma), \quad \text { near }\{t=0\} .
\end{gathered}
$$

with

$$
\gamma=\Omega \times\left\{(\xi, \omega) \in \mathbb{R}^{n+1}, \xi_{n}^{2}+\left|\xi^{\prime}\right|^{2}=\omega^{2} \text { and }\left|\xi_{n}\right| \leq \epsilon_{0}\left|\xi^{\prime}\right|\right\}
$$

for small $\epsilon_{0}>0 . \Omega$ is a compact subset of $\left\{(x, t) \in \mathbb{R}^{n+1},|t|,\left|x_{n}\right| \leq \epsilon_{0}\right\}$ and

$$
\Gamma=\text { a conic neighborhood of } \gamma \text {. }
$$

Assume that

(i) $u \in H^{l-1} \cap H_{m \ell}^{l}(\Gamma), 1 \leq l \leq s$;

(ii) $\nabla \sigma(x) \in H^{s-1} \cap H_{m \ell^{(}}^{l}(K), \Pi: \Gamma \subset T^{*}\left(\mathbb{R}^{n+1}\right) \rightarrow K \subset T^{*}\left(\mathbb{R}^{n}\right)$ is the projection map;

(iii) $f \in H^{l-1} \cap H_{m \ell}^{l}(\Gamma)$.

Then

$$
\left.u\right|_{x_{n}=0} \in H_{l o c}^{l}
$$

in fact,

$$
\left\|\left.(\phi u)\right|_{x_{n}=0}\right\|_{l} \leq C_{1}\left\|\phi_{0} u\right\|_{l}+C\left\|\phi_{0} f\right\|_{l-1}+C\left\|\phi_{1} P \phi_{0} f\right\|_{l}
$$

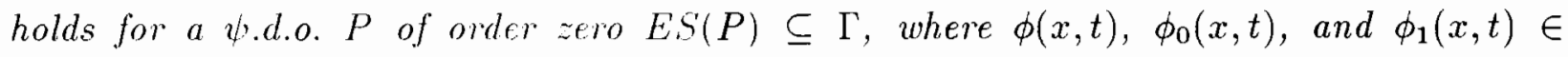
$C_{0}^{\infty}\left(\mathbb{R}^{n+1}\right)$ are supported near the trace hypersurface $\left\{x_{n}=0\right\}$ and the constant $C_{1}$ depends 
on $H^{s-1} \cap H_{m \ell}^{l}(K)$-norm of $\psi \nabla \sigma$ with $\psi \in C_{0}^{\infty}\left(\mathbb{R}^{n}\right)$ and $\operatorname{supp}(\phi)$, supp $\left(\phi_{1}\right) \subset \operatorname{supp}\left(\phi_{0}\right)$, $\phi_{0}>0$ on $\operatorname{supp}(\phi)$ and $\operatorname{supp}\left(\phi_{1}\right)$

Idea of the proof. As we mentioned before, since the hypersurface $\left\{x_{n}=0\right\}$ is a time-like surface, the method of energy estimates cannot be applied directly. To cure this difficulty, we shall alter the wave operator $\square$ by a $\psi$.d.o. cutoff technique so that $\left\{x_{n}=0\right\}$ becomes a space-like surface. In other words, we shall construct a strictly hyperbolic $\psi . d . o$. equation with respect to the trace $\left\{x_{n}=0\right\}$. Since the operator in our construction is differential in $x_{n}$, the standard method of energy estimates (for example in John [9] or Taylor [18]) can be applied to get the basic estimate. Then, the microlocal hypotheses and Theorem 2.1 together with Lemmas $3.1,3.2$ will complete the proof.

Proof. Let $\gamma_{0}, \gamma_{1}$ be two conic subsets of the set $\Omega_{0} \times\left\{\left(\xi^{\prime}, \omega\right) \in \mathbb{R}^{n},|\omega| \geq\left|\xi^{\prime}\right|\right\}$ and let $\Pi_{2} \gamma_{0}^{c l} \subset \Pi_{2} \gamma_{1}^{\text {int }}$, where $\Pi_{2}$ maps a set to its second factor or the frequency space, and $\Omega_{0}$ is generated by $\Omega$ in such a way that each point in $\Omega_{0}$ may be traced back to $\Omega$ along the characteristics of the operator $\square$. That is,

$$
\Omega_{0}=\left\{(x, t) \in \mathbb{R}^{n+1}, \exists\left(x_{0}, t_{0}\right) \in \Omega, x=x_{0}-\lambda t,|\lambda|=1\right\}
$$

Then, we can find a convolutional operator $Q \in C^{\infty}\left(\mathbb{R}, O P S^{0}\left(\mathbb{R}^{n}\right)\right), q=q\left(\xi^{\prime}, \omega\right)$, that satisfies

- $\operatorname{ES}(Q) \subset \gamma_{1}$ and $0 \leq Q_{0} \leq 1$;

- $Q_{0}=1$ on $\gamma_{0} \cap\left\{\left(x, t, \xi^{\prime}, \omega\right),\left|\left(\xi^{\prime}, \omega\right)\right|>1\right\}$,

where $Q_{0}\left(\xi^{\prime}, \omega\right)$ is the principal symbol of $Q$. Define another operator $E$ as

$$
E \stackrel{d E S .}{=} Q \square_{x^{\prime}, t}+(I-Q) \Delta_{x^{\prime}, t}
$$


where $\square_{x^{\prime}, t}=\partial_{t}^{2}-\partial_{x^{\prime}}^{2}$ and $\Delta_{x^{\prime}, t}=\partial_{t}^{2}+\partial_{x^{\prime}}^{2}$.

Observe that the principal symbol of $E$

$$
E_{0}=Q_{0}\left(\omega^{2}-\left|\xi^{\prime}\right|^{2}\right)+\left(1-Q_{0}\right)\left(\omega^{2}+\left|\xi^{\prime}\right|^{2}\right) \geq C\left(\omega^{2}+\left|\xi^{\prime}\right|^{2}\right)
$$

for $\left|\left(\omega, \xi^{\prime}\right)\right| \geq \delta$, with some positive constants $C, \delta$. Hence, $E$ is an elliptic $\psi$.d.o. of order two.

Let $\phi=\phi(x, t) \in C_{0}^{\infty}\left(\mathbb{R}^{n+1}\right)$ with supp $\phi \subseteq\left\{\left|x_{n}\right| \leq \epsilon_{0}\right\}$. We then have a strictly symmetric hyperbolic problem

$$
\begin{aligned}
\left(-\partial_{x_{n}}^{2}+E\right) \phi u & =\square \phi u+(I-Q)\left(\Delta_{x^{\prime}, t}-\square_{x^{\prime}, t}\right) \phi u \\
& =[\square, \phi] u+\phi f+\phi \nabla \sigma \cdot \nabla u+(I-Q)\left(\Delta_{x^{\prime}, t}-\square_{x^{\prime}, t}\right) \phi u
\end{aligned}
$$

Since $\phi$ is compactly supported, we actually have a symmetric hyperbolic Cauchy problem with zero Cauchy data with $x_{n}$ playing the role of "time". It follows from a hyperbolic energy estimate in Taylor $[18]$ pages $73-75$, by knowing that $[\square, \phi]$ and $\left[\Delta_{x^{\prime}, t}-\square_{x^{\prime}, t}\right]$ are operators of order one and two respectively, that

$$
\begin{aligned}
\left\|\left.(\phi u)\right|_{x_{n}=0}\right\|_{l} & \leq C \| r . h . s \text { of }(3.4) \|_{l-1} \\
& \leq C\left[\left\|\phi_{1} u\right\|_{l}+\|\phi f\|_{l-1}+\|\phi \nabla \sigma \cdot \nabla u\|_{l-1}+\left\|(I-Q) \partial_{x^{\prime}}^{2} \phi u\right\|_{l-1}\right],
\end{aligned}
$$

where $\phi_{0} \in C_{0}^{\infty}$, and the second inequality makes sense because $Q$ is a convolutional operator.

From the hypotheses, a natural extension of the Proposition in Beals and Reed [6] pages 176-177 leads to the fact that $u \in H_{l u c}^{l}$. Thus, the generalized Schauder's lemma (Lemma 2.2) yields

$$
\phi \nabla \sigma \cdot \nabla u \in H^{l-1} .
$$


Therefore, to complete the proof it suffices to show that

$$
(I-Q) \partial_{x^{\prime}}^{2} \phi u \in H^{l-1},
$$

which requires the use of Lemma 3.1. In order to apply Lemma 3.1, we choose $B=I-Q \in$ $C^{\infty}\left(\mathbb{R}^{1}, O P S^{0}\left(\mathbb{R}^{n}\right)\right)$ of order $m=0, A=\square$ of order $m_{0}=2$, and $h=l-2$ in the statement of Lemma 3.1.

Let us look at the assumption (1) of Lemma 3.1, Ell( $A$ ) (the elliptic region of $A=\square$ ) is easy to determine. Actually $\square$ is elliptic away from the light cone $\left\{\omega^{2}=|\xi|^{2}\right\}$. To verify hypothesis (3), one only needs to look at

$$
\square \partial_{x^{\prime}}^{2} \phi u=\left[\square, \partial_{x^{\prime}}^{2} \phi\right] u+\partial_{x^{\prime}}^{2} \phi(\nabla \sigma \cdot \nabla u+f),
$$

which is bounded by the first three terms in (3.2); hence the same arguments yield that $\square \partial_{x^{\prime}}^{2} \phi u \in H^{l-3}$. Therefore, the only assumption that needs to be checked is that

$$
u \in H_{m !}^{l+1}\left(\left[T^{* *}\left(\mathbb{R}^{n+1}\right) \backslash E l l(\square)\right] \cap \tilde{\Pi}_{2}^{-1} E S(I-Q)\right)
$$

and this demands the Beals-Reed type theorem on propagation of singularities (Theorem 2.1) and Lemma 3.2.

In the statement of Theorem 2.1 choose

$$
\left(m, n_{0}, n, l, s, q, \epsilon\right)=(2, n, n+1, l-1, s-1, l, 1)
$$

then the microlocal hypotheses verify all the assumptions of Theorem 2.1. Notice that the main assumption, $s>3+n / 2$, is required by the corresponding hypothesis (i) in Theorem 2.1. Let $\gamma_{0}$ and $\gamma_{1}$ approach the set $\Omega_{0} \times\left\{(\xi, \omega):|\omega| \geq\left|\xi^{\prime}\right|\right\}$. The set

$$
\left[T^{*}\left(\mathbb{R}^{n+1}\right) \backslash E l l(\square)\right] \cap \tilde{\Pi}_{2}^{-1} E S(I-Q)
$$


is contained in a small (conic) neighborhood of the Hamiltonian flow out of $\gamma$. Hence Theorem 2.1, Lemma 3.2 (in particular, the remarks there), and the microlocal initial hypotheses yield that

$$
u \in H_{m \ell}^{l+1}\left(\left[T^{* *}\left(\mathbb{R}^{n+1}\right) \backslash E l l(\square)\right] \cap \tilde{\Pi}_{2}^{-1} E S(I-Q)\right) .
$$

It follows from Lemma 3.2 that there exists a $\psi$.d.o. $P$ of order zero such that

- $P$ is elliptic on $\left.\left[T^{*}\left(\mathbb{R}^{n+1}\right) \backslash E l l(\square)\right] \cap \tilde{\Pi}_{2}^{-1} E S(I-Q)\right)$;

- $[\square, P] \in O P S^{0}$;

- $p$ is supported near $\left\{x_{n}=0\right\}$.

From the ellipticity of $P$, Lemma 3.1'and Gårding's type inequality Lemma 3.3 yield that

$$
\begin{aligned}
\left\|(I-Q) \partial_{x^{\prime}}^{2} \phi u\right\|_{l-1} & \leq C\left\|\phi_{1} P \partial_{x^{\prime}}^{2} \phi u\right\|_{l-1}+C\|\phi u\|_{r} \\
& \left.\leq C\|\| \phi_{1} P, \partial_{x^{\prime}}^{2}\right] \phi u\left\|_{l-1}+C\right\| \phi_{1} P \phi u\left\|_{l+1}+C\right\| \phi u \|_{r} \\
& \leq C\left\|\phi_{0} u\right\|_{l}+C\left\|\phi_{1} P \phi u\right\|_{l+1}+C\|\phi u\|_{r}
\end{aligned}
$$

for any $r \in \mathbb{R}$. Thus the proof has been reduced to bounding $\left\|\phi_{1} P \phi u\right\|_{l+1}$.

Applying $\phi_{1} P \phi$ on both sides of equation (3.1), one has

$$
\square \phi_{1} P \phi u=\left[\square, \phi_{1} P \phi\right] u+\phi_{1} P \phi \nabla \sigma \cdot \nabla u+\phi_{1} P \phi f .
$$

Then the energy estimates together with a simple estimate implied by the commutator lemma (Lemma 2.4) yield

$$
\left\|\phi_{1} P \phi u\right\|_{l+1} \leq C\left\|\phi_{0} u\right\|_{l}+C\left\|\phi_{0} f\right\|_{l-1}+C\left\|\phi_{0} P \phi f\right\|_{l},
$$


where $C$ depends on the $H^{s-1} \cap H_{m \ell}^{l_{1}}\left(K^{\prime}\right)$-norm of $\psi \nabla \sigma$. Here we have used the fact that $\phi_{1}(x, t) \in C_{0}^{\infty}\left(\mathbb{R}^{n+1}\right)$ or $\phi_{1}(x, t)$ has compact support in $t$.

The proof of Theorem 3.1 is then completed.

Two remarks on Theorem 3.1 are in order:

- Hypothesis (3.2) requires additional microlocal regularity near $t=0$, as demanded by the application of the result on propagation of singularities. This hypothesis is rather difficult to verify in practice. It seems that similar hypotheses are made in all the previous results on propagation of singularities. We believe that with the help of a duality argument Hypothesis (3.2) can be replaced by a hypothesis on microlocal regularity of the Cauchy data on $\{t=0\}$. Similar (but more subtle) approach may be found in Bao and Symes [3]. Details of this and other related issues will be addressed elsewhere.

- We conjecture that the regularity assumptions on the coefficients can not be strengthened, see our remark on Theorem 2.1. The fact that the local regularity assumption on $f$ is optimal is evident. However, it still remains to see that whether the microlocal regularity requirement on $f$ is optimal.

\section{Acknowledgement}

We would like to thank Professor Irena Lasiecka and an anonymous referee for their constructive suggestions. 


\section{References}

[1] G. Bao, Microlocal regularity of an inverse problem for the multidimensional wave equation, Ph.D. thesis, Technical report 90-34, Dept. of Math. Sci., Rice Univ., Houston, Texas (1990).

[2] G. Bao and W. Symes, A trace theorem for solutions of linear partial differential equations, Math. Meth. in the Appi. Sci., Vol. 14 (1991), 553-562.

[3] G. Bao and W. Symes, An upper bound for the linearized map of an inverse problem for the wave equation, Technical report 91-2, Dept. of Math. Sci., Rice Univ., Houston, Texas (1991).

[4] M. Beals, Propagation of smoothness for nonlinear second order strictly hyperbolic equations, Proc. of Symp. in Pure Math. 43 (1985), 21-44.

[5] M. Beals, Propagation and Interaction of Singularities in Nonlinear Hyperbolic Problems, Birkhäuser, Boston, 1989.

[6] M. Beals and M. Reed, Propagation of singularities for hyperbolic pseudodifferential operators and applications to nonlinear problems, Comm. Pure Appl. Math. 35 (1982), $169-184$.

[7] M. Beals and M. Reed, Microlocal regularity theorems for nonsmooth pseudodifferential operators and applications to nonlinear problems, Trans. Am. Math. Soc. 285 (1984), $159-184$. 
[8] L. Hörmander, Linear differential operators, Actes Congr. Internat. Math., Vol.1 (Nice, 1970). Gauthier-Villars, Paris (1971), 121-133.

[9] F. John, Partial Differential Equations, Springer-Verlag, New York, 1982.

[10] I. Lasiecka and R. Triggiani. Trace regularity of the solutions of the wave equation with homogeneous Neumann boundary conditions and compactly supported data, J. Math. Anal. and Appl., 14 (1989), 49-71.

[11] I. Lasiecka and R. Triggiani. Sharp regularity theory for second order hyperbolic equations of Neumann type, Annali di Matem. Pure et Applic. (IV) Vol. CLVII (1990).

[12] Y. Meyer, Régularité des solutions des équations aux derivées partielles nonlinéaires, Sem. Bourbaki, No. 560 (1979-1980).

[13] L. Nirenberg, Lectures on Linear Partial Differential Equations, CBMS Regional Conf. Ser. in Math., No. 17, Amer. Math. Soc., Providence, R.I., 1973.

[14] J. Rauch, Singularities of solutions to semilinear wave equations, J. Math. Pures Appl. 58 (1979), 299-308.

[15] J. Rauch and M. Reed, Nonlinear microlocal analysis of semilinear hyperbolic systems in one space dimension, Duke Math. J., 49 (1982), 379-475.

[16] W. Symes, A trace theorem for solutions of the wave equation, and the remote determination of acoustic sources, Math. Meth. in the Appl. Sci. 5 (1983), 131-152. 
[17] M. Taylor, Reflection of singularities of solutions to systems of differential equations, Comm. on Pure and Appl. Math. 28 (1975), 457-475.

[18] M. Taylor, Pseudo-Differential Operators, Princeton Univ. Press, Princeton, N.J., 1981. 